\title{
Nanocomputing in medicine
}

\begin{abstract}
The need for protecting and maintaining the confidentiality of sensitive data has become of great importance and concern now a days due to the rapid growth of internet and fast information exchange technologies. In medicine it is of great concern to store the integrity of the personal medical data especially in the form of images. The emerging field of DNA based nanocomputing methods offers a relief to this problem which utilizes DNA sequences to hide data. The paper presents a review on the concept of DNA cryptography and DNA computing in general. It also presents an overview of various possible applications of DNA based nanocomputing.
\end{abstract}

Keywords: nanocomputing, medical imaging, DNA cryptography, nano devices, quantum dots
Volume 3 Issue | - 2018

\author{
Smrithi S, Supriya R, Neethu Hari, A \\ Jayakumaran Nair \\ Department of Biotechnology, University of Kerala, India
}

Correspondence: Supriya R, Department of Biotechnology, University of Kerala, Kariavattom Campus, Thiruvananthapuram, 69558I, India, Email supriyaradhakrishnan@gmail.com

Received: March 28, 2018 | Published: April 30, 2018
Abbreviations: CMOS, complementary metal oxide semiconductor; WHO, world health organization; LMWP, low molecular weight proteome

\section{Introduction}

Nanotechnology was introduced to the world by Richard Feynman in 1959 through the talk "There is plenty of room at the bottom". From the day onwards the use of molecules below the micrometer dimensions have found its use across many fields. The change in structural and chemical features of molecules when they are reduced to their nanoscale sizes have given rise to the prospect of developing materials and devices with new enhanced properties capable of doing much more than their bulk counterpart. Inevitably the use of nanotechnology has also found its use in computer science and could be the first step towards creating supercomputers that could fit inside one's palm. Nanocomputing is the use of submicron devices for solving computational problems. ${ }^{1}$ In the current scenario, processors, an integral part of modern day computers, have been fabricated using semiconductor integrated circuits built with silicon using the technique of complementary metal oxide semiconductor (CMOS). ${ }^{2}$ Miniaturization of silicon based transistors could lead to an increase in power consumption, heat dissipation and higher manufacturing expense. Through its use, nanotechnology looks to overcome the problems of current computing techniques with advantages such as miniaturization, parallelism, high processing ability, larger memory and speed. Low cost and the need for lesser area. Nanocomputing could also be based on molecules such as DNA or proteins. DNA is more probable choice due to its inherent properties such as its unique double helix structure, hybridization and regeneration capabilities through which it can be employed to find solutions to highly complex computational problems.

\section{Uses of Nanocomputing in medicine}

In the field of medicine, storage of personal medical data is very difficult. The confidentiality, integrity, reliability and security in storage and transmission of digital images are the primary concerns in the medical field. ${ }^{3}$ Through DNA based nanocomputing this problem can be solved to some extent. Using Chen's hyper chaotic map and the Lorenz chaos system it is able to enhance the security of the medical images. ${ }^{4}$ Since the DNA sequences are very complex and complicated to predict and analyze for each individual it is suitable to enhance the security of medical image encryption and provides high security required for digital medical images. The use of nanotechnology in computing is becoming a potential alternative and its applications in the medicinal field are also investigated worldwide. Nanocomputing could be used in the development of biosensors capable of detection of disease or abnormalities, body implantable devices capable of targeted drug release and precise imaging of internal organs. ${ }^{5}$ Targeted drug delivery using magnetic nanoparticles could effectively fight off diseases localized in certain tissues without affecting the entire body.

With the direct correlation between early diagnosis and survival rate, nanocomputing could be used in bringing down mortality rate caused by microbial diseases by making prior detection possible. ${ }^{6}$ According to the report by World Health Organization (WHO), nearly 50,000 people including children die every day due to infections. Much of it could be reduced by early diagnosis and treatment. Usual diagnosing techniques require weeks for confirmation of diseases whereas nanodevices could make diagnosis possible within 24 hours which is beneficial especially in cases of infections like tuberculosis that requires earliest detection for better treatment. ${ }^{7}$ Nanodevices can also be employed to detect the protein levels of the serum such as the low molecular weight proteome (LMWP) that could be an efficient indicator of various pathological conditions. Quantum dots associated with antibodies based immunoassays are also arising as technique for multiplex detection of diseases. Quantum dots also finds potential use as less toxic alternative to organic dyes used in medical imaging. ${ }^{6}$ Nanotechnology based biosensors is presently investigated as a means for characterization of diseases by allowing real time sensing and monitoring of various biomolecules such as hormones, growth factors, enzymes. ${ }^{8}$ and even RNA molecules that have a key role in regulating gene expression such as the micro RNA without any toxic labelling. Various lifestyle diseases such as diabetes could be effectively managed by the use of biosensors. ${ }^{6}$

\section{Conclusion}

Nanotechnology, within its smaller dimensions, holds great potential in improving the quality of human life by enabling faster multiplex detection and targeted drug delivery to manage and cure 
diseases. When developed to its utmost potential, nanotechnology could enable cheaper yet effective treatment to the masses and could revolutionize the medicinal industry. Though nanocomputing is in its embryonic stage yet, DNA based nanocomputing is a growing area of research which offers wide applications in forensics and medicine.

\section{Acknowledgments}

None.

\section{Conflict of interest}

Authors have declared no conflict of interests.

\section{References}

1. Deepti H, Anuradha S. Nanocomputing revolution and future prospects. Recent Research in Science and Technology. 2012;4(3): 16-17.

2. Saha H, Chaudhari C. Complementary Metal Oxide Semiconductors Microelectromechanical Systems Integration. Defence Science Journal. 2009;59(6):557-567.
3. Nikita P, Ashwin N. Survey of different DNA Cryptography based algorithms. International Research Journal of Engineering and Technology. 2017;4(12):1-5.

4. Jyoti C, Anchal J. Survey On Encryption Algorithm Based On Chaos Theory And DNA Cryptography. International Journal of Advanced Research in Computer and Communication Engineering. 2014;3(8):1-3.

5. Sujatha S. Advances in Computer Science and Information Technology. 2015;(7):53-57.

6. Ye Hu, Daniel H, Fine, et al. Nanodevices in diagnostics. Wiley Interdiscip Rev Nanomed Nanobiotechnol. 2011;3(1):11-32.

7. Wang Y, Li Yu, Kong X, et al. Application of nanodiagnostics in point-ofcare tests for infectious diseases. Int J Nanomedicine. 2017;12:4789-4803.

8. Prasad S. Nanobiosensors: future for diagnosis of disease? Nanobiosensors in Disease Diagnosis. 2014;3:1-10. 